\title{
L'ammissione della prova in appello tra diritto di difesa e parità delle parti
}

\author{
The admission of the evidence in appeal between \\ right of defence and equality of arms
}

\author{
Lorenzo Agostino ${ }^{1}$ \\ Università degli Studi di Genova, Italia \\ lorenzo.agostino@edu.unige.it \\ https://orcid.org/0000-0002-6132-5402
}

\begin{abstract}
AвSTRACT: Il tema dell'ammissione delle prove in appello rappresenta un tipico terreno di scontro tra l'esigenza di assicurare la ragionevole durata dei processi e la necessità di rispettare, anche in sede di secondo grado, i principi del giusto processo. II presente lavoro si propone di analizzare le insoddisfacenti soluzioni adottate nel corso del tempo dal legislatore italiano al fine di contemperare questi opposti interessi e di evidenziare l'opportunità di un ripensamento del mezzo di impugnazione, ancora strutturato in chiave inquisitoria nonostante l'impostazione tendenzialmente accusatoria del codice di rito.

Parole ChiAve: Ammissione delle prove; Appello; Rinnovazione istruttoria; Parità delle parti; Equo processo.
\end{abstract}

ABSTRACT: The issue of the admission of the evidences in appeal represents a typical battleground between the interest in ensuring the reasonable duration of trials and the opportunity to respect, even in appeal, the fair trial's principles. The paper aims to analyse the unsatisfactory solutions adopted over the years by the Italian legislator in order to reconcile these opposite needs and to emphasize the importance of a reform of the remedy, still structured in an inquisitorial way despite the accusatory setting of the code of criminal procedure.

KeYwords: Admission of evidences; Appeal; Renewal of the trial evidentiary hearing; Equality of arms; Fair trial.

1 Dottorando di ricerca in Diritto processuale penale, Università degli Studi di Genova. 
Sommario: Premessa; 1. Gli angusti spazi del diritto alla prova in appello; 2 . La questione del ribaltamento in appello della sentenza di proscioglimento; 3. L'art. 603 comma 3-bis tra diritto di difesa e parità delle parti; 4. L'ammissione delle prove dichiarative in caso di appello dell'imputato avverso la sentenza di condanna; Considerazioni conclusive; Bibliografia.

\section{Premessa}

La giustizia penale è caratterizzata da una costante tensione tra la necessità di assicurare all'accusato il diritto di difendersi nei confronti dell'imputazione elevata a suo carico e la salvaguardia dell'efficienza di un sistema che, senza adeguate contromisure, sarebbe destinato al collasso.

Uno dei settori in cui l'attrito tra questi opposti interessi è senz'altro evidente è quello delle impugnazioni che, seppur finalizzate a garantire l'adozione della decisione più corretta, sono contraddistinte dall'individuazione di inevitabili limitazioni concernenti anche il diritto alla prova, di cui le parti usufruiscono ad ampio raggio in primo grado. Un sistema già "ingolfato" qual è quello italiano, infatti, non sopporterebbe un mezzo di gravame che consentisse di ripetere ex novo tutta l'attività svolta di fronte al primo giudice ${ }^{2}$.

Dinanzi al legislatore si pone quindi da sempre la non semplice sfida di disegnare i confini entro i quali contenere l'attività istruttoria in sede di appello, compito da assolvere senza perdere di vista quei principi espressi tanto dalle fonti sovrannazionali, quanto dalla Costituzione - che, riguardando il processo tout court, devono essere rispettati in tutti i gradi di cui esso si compone.

2 D'altronde, «il tempo da dedicare al contraddittorio impegna a fondo la struttura del processo, diventando elemento capace di dilatarne la durata», con la conseguenza che si impone «un avveduto bilanciamento tra garanzie ed efficienza» (BELLUTA, Hervé. Prospettive di riforma dell'appello penale: tra modifiche strutturali e microchirurgia normativa. In: BARGIS, Marta, BELLUTA, Hervé. Impugnazioni penali. Assestamenti del sistema e prospettive di riforma. Torino: Giappichelli, 2013, p. 247). 
Nelle pagine seguenti si tenterà quindi di analizzare se le scelte operate in questo campo dal legislatore italiano siano o meno soddisfacenti.

\section{GLI ANGUStI SPAZI DEL DIRITTO ALLA PROVA IN APPELLO}

Tra le due esigenze di cui si è detto poc'anzi, nella disciplina del giudizio di appello è per lungo tempo prevalsa quella di natura efficientistica, considerato il disinteresse mostrato dal legislatore del 1988 nei riguardi di un istituto che ha per gran parte ereditato la normativa del codice di rito del 1930, benché quest'ultimo fosse ispirato a una modellistica di matrice inquisitoria ${ }^{3}$.

La similitudine tra i giudizi di secondo grado contemplati dai due corpus normativi «appare manifest[a] e viene consacrat[a]» proprio dall'art. 603 c.p.p. che, nel disciplinare la rinnovazione istruttoria in appello, ricalca l'art. 520 c.p.p. abr. ${ }^{4}$.

Difatti, al di là del diritto di ottenere l'ammissione a "maglie larghe", cioè sulla base degli stessi ampi presupposti previsti in primo grado, delle prove scoperte o sopravvenute dopo la pronuncia della decisione impugnata, l'art. 603 c.p.p., fatto salvo il "nuovo" comma 3-bis dota la corte d'appello del potere di scegliere discrezionalmente se e in che misura riassumere le fonti dichiarative ${ }^{6}$.

3 La scarsa attenzione rivolta nei confronti dell'appello emerge con chiarezza già dalla Relazione al progetto preliminare del codice di procedura penale, in G.U., 24 ottobre 1988, n. 250, Suppl. ord. n. 2, p. 124, da cui risulta come in sede di lavori preparatori non abbia avuto luogo alcun dibattito tanto dal punto di vista della configurazione dei mezzi di impugnazione come strumenti per riparare gli errori contenuti nella decisione impugnata o per controllare l'operato del giudice, quanto sul piano dell'opportunità o meno di mantenere il generale criterio dell'appellabilità delle decisioni.

4 Così ZAPPALÀ, Enzo. Sub art. 603. In: CHIAVARIO, Mario (coordinato da). Commento al nuovo codice di procedura penale, vol. VI. Torino: Utet, 1991, p. 201.

5 V. infra $\S 4$.

6 Sotto questo profilo, la giurisprudenza si è sempre mostrata molto rigida nell'interpretazione delle clausole della non decidibilità allo stato degli atti e della assoluta necessità cui rispettivamente i commi 1 e 3 della menzionata disposizione subordinano lo svolgimento di attività probatoria. Sul punto 
Così, sin da subito la dottrina italiana ha avuto modo di evidenziare la «contraddizione» insita in un sistema che ad un primo grado, in cui le prove sono assunte «alla stregua del principio dell'oralità e del contraddittorio nel momento della formazione della prova», giustappone un appello «basato sugli atti del processo», con il conseguente rischio che la sentenza sia riformata «da un giudice davanti al quale non è stata assunta nessuna prova» ${ }^{7}$. Tale incongruenza è stata vieppiù acuita dalla revisione dell'art. 111 Cost., il cui quarto comma stabilisce che il processo penale - espressione idonea a ricomprendere anche il giudizio di seconda istanza ${ }^{8}$ - è "illuminato" «dal principio del contraddittorio nella formazione della prova».

Questo attrito tra l'impianto accusatorio del processo e le caratteristiche del rimedio potrebbe in futuro interessare anche l'ordinamento brasiliano, per due ordini di motivi: da un lato, al pari del giudice d'appello italiano, anche quello sudamericano domina la materia probatoria, dato che, ai sensi dell'art. 616 c.p.p., «no julgamento das apelações poderá o tribunal, câmara ou turma proceder a novo interrogatório do acusado, reinquirir testemunhas ou determinar outras diligências» ${ }^{9}$; dall'altro, il Brasile ha intrapreso un percorso volto al superamento della logica inquisitoria sottesa ad un codice che, adottato nel 1941, ha recepito «as influências do Código Rocco, de forte inspiração fascista» ${ }^{10}$. Infatti, dopo l'entrata in vigore della Constituição del 1988, che già aveva fatto propria la regola del contraddittorio, la lei 24 dicembre 2019, n. 13.964, ha introdotto, all'interno dell'art. $3^{\circ}$-A c.p.p., il principio - la cui efficacia

cfr., ex multis, Cass., sez. IV, 23 maggio 2017, n. 8106, in Diritto \& Giustizia, 6 luglio 2017.

7 LOZZI, Gilberto. Lezioni di procedura penale. $12^{\mathrm{a}}$ ed. Torino: Giappichelli, 2017, p. 697-698.

8 A tale proposito si veda, diffusamente, CHINNICI, Daniela. Giudizio penale di seconda istanza e giusto processo. Torino: Giappichelli, 2009, p. 39 ss.

9 Infatti, secondo GRINOVER, Ada Pellegrini, MAGALHÃES, Antonio, SCARANCE, Antonio. Recursos no Processo Penal. 5a ed. São Paulo: RT, 2008, p. 53, «dentro da matéria impugnada, são plenos os poderes instrutórios do tribunal, ainda que os códigos somente se refiram a eles para certos recursos».

10 Così GIACOMOLLI, Nereu José. Algumas marcas inquisitoriais do Código de Processo Penal brasileiro e a resistência às reformas. Revista Brasileira de Direito Processual Penal, Porto Alegre, vol. 1, n. 1, p. 144, 2015. 
invero è al momento sospesa - secondo cui «o processo penal terá estrutura acusatória, vedadas a iniciativa do juiz na fase de investigação e a substituição da atuação probatória do órgão de acusação» ${ }^{11}$.

Ebbene, l'ambiguo rapporto tra i primi due gradi di giudizio cui dà luogo la normativa italiana ha posto (e continua a porre) due questioni di grande rilievo: una di carattere epistemologico, legata all'eventualità di una modifica della sentenza di primo grado nell'ambito di un giudizio, quello di appello, dai connotati sostanzialmente opposti; l'altra, che invero è una sottocategoria della prima, attinente più specificamente al diritto di difesa dell'imputato, che in sede di gravame corre il pericolo di una riforma peggiorativa della prima decisione.

È su quest'ultimo profilo che si è primariamente appuntata l'attenzione del legislatore e della giurisprudenza, tanto europea quanto interna.

\section{LA QUESTIONE DEL RIBALTAMENTO IN APPELLO DELLA SENTENZA DI PROSCIOGLIMENTO}

La più evidente distorsione derivante dall'illustrata conformazione del giudizio d'appello è rappresentata dalla concreta possibilità che l'imputato, prosciolto in primo grado, venga condannato al termine del secondo giudizio a seguito della mera rilettura dei verbali contenuti nel fascicolo dibattimentale.

Il rimedio, inizialmente architettato dal legislatore con la 1. 20 febbraio 2006, n. 46, nota anche come "legge Pecorella", fu radicale: lasciando inalterati gli angusti spazi riservati all'attività istruttoria, la riforma si concentrò sull'appellabilità “oggettiva” delle sentenze, con l'abolizione del potere del pubblico ministero e dell'imputato di appellare le pronunce di proscioglimento ${ }^{12}$.

11 Ad opinione di LOPES JR., Aury. Direito processual penal. 17ª ed. São Paulo: Saraiva Educação, 2020, p. 71, grazie a queste riforme «agora podemos afirmar que o processo penal brasileiro é legal (art. $3^{\circ}$-A do CPP) e constitucionalmente acusatório», sebbene "para efetivação dessa mudança é imprescindível afastar a vigência de vários artigos do CPP e mudar radicalmente as práticas judiciárias».

12 Per un commento alla riforma si vedano, tra gli altri, BARGI, Alfredo. I nuovi ambiti oggettivi delle impugnazioni. In: GAITO, Alfredo (a cura di). La 
Al di là del fatto che questo intervento non eliminava completamente la possibilità del ribaltamento della decisione liberatoria ${ }^{13}$, la novella ebbe vita breve, dal momento che la Corte costituzionale ne dichiarò l'illegittimità data l'irragionevole disparità di trattamento che la medesima aveva prodotto tra la posizione dell'accusa, impossibilitata ad appellare le sentenze che la vedevano "soccombente", cioè quelle assolutorie, e la difesa che, al contrario, rimaneva legittimata ad esperire il gravame avverso la condanna ${ }^{14}$.

Il fallimento della legge del 2006 ha riproposto il problema, stavolta affrontato - sulla base dell'impulso proveniente dalla Corte europea dei diritti dell'uomo - sotto un diverso angolo visuale, quello dell'ammissione delle prove nel giudizio di gravame.

I Giudici di Strasburgo, seppur con i limiti del loro tipico approccio casistico, hanno elaborato alcune garanzie volte a preservare l'equità processuale laddove si tratti di procedere ad un overturning della sentenza di proscioglimento in virtù di una rivalutazione delle affermazioni rese da una fonte dichiarativa nel corso del giudizio di primo grado ${ }^{15}$. È stato

nuova disciplina delle impugnazioni dopo la "legge Pecorella". Torino: Utet, 2006, p. 151 ss.; MARANDOLA, Antonella. Nuovo regime dei casi d'appello. In: SCALFATI, Adolfo (a cura di). Novità su impugnazioni penali e regole di giudizio. Trento: Ipsoa, 2006, p. 121 ss.; PRESUTTI, Adonella. L'inappellabilità delle sentenze di proscioglimento tra regola ed eccezione. In: BARGIS, Marta, CAPRIOLI, Francesco (a cura di). Impugnazioni e regole di giudizio nella legge di riforma del 2006. Dai problemi di fondo ai primi responsi costituzionali. Torino: Giappichelli, 2007, p. 51 ss.

13 Difatti, residuava la possibilità di appellare la sentenza di proscioglimento in presenza di una prova nuova o sopravvenuta che fosse stata decisiva.

14 Cfr. Corte cost., 24 gennaio 2007 (dep. 6 febbraio 2007), n. 26, in Giurisprudenza costituzionale, p. 221, 2007 con nota di CAPRIOLI, Francesco. Inappellabilità delle sentenze di proscioglimento e "parità delle armi" nel processo penale.

15 Tra le varie pronunce che si sono susseguite in materia ricordiamo: Corte edu, Gr. Cam., 26 maggio 1988, Ekbatani c. Svezia, ricorso n. 10563/83; Corte edu, II sez., 18 maggio 2004, Destrehem c. Francia, ricorso n. 56651/00; Corte edu, III sez., 5 luglio 2011, Dan c. Moldavia, ricorso n. 8999/07; Corte edu, III sez., 5 marzo 2013, Manolachi c. Romania, ricorso n. 36605/04; Corte edu, III sez., 4 giugno 2013, Hanu c. Romania, ricorso n. 10890/04. Per quanto riguarda l'ordinamento italiano, v. Corte edu, I sez., 29 giugno 2017, Lorefice c. Italia, ricorso n. 63446/13, nonché Corte edu, I sez., 22 ottobre 2020, Tondo c. Italia, ricorso n. 75037/14. Queste pronunce sono tutte disponibili 
in particolare affermato come, in linea di principio ${ }^{16}$, coloro che hanno la responsabilità di decidere sulla colpevolezza o l'innocenza dell'imputato debbano sentire personalmente i testimoni e saggiarne la credibilità, dal momento che «la valutazione dell'attendibilità di un testimone è un'attività complessa che, normalmente, non può essere svolta mediante una semplice lettura del contenuto delle dichiarazioni di quest'ultimo, come riportate nei verbali delle audizioni» ${ }^{17}$.

Data la refrattarietà del giudizio d'appello italiano a consentire lo svolgimento di attività istruttoria ${ }^{18}$, la questione non ha tardato a spostarsi dai tavoli della Corte di Strasburgo a quelli della Corte di cassazione, in seno alla quale sono emersi non pochi contrasti, che hanno richiesto per ben tre volte l'intervento chiarificatore delle Sezioni Unite.

Il fil rouge che unisce queste pronunce è rappresentato dalla valorizzazione del principio, espresso dall'art. 533 c.p.p. in tema di

all'indirizzo https://hudoc.echr.coe.int/. Per un'analisi delle sentenze della Corte europea in materia si vedano TESORIERO, Salvatore. La rinnovazione della prova dichiarativa in appello alla luce della CEDU. Diritto penale contemporaneo, Rivista trimestrale, v. 3-4, p. 239-276, 2014; AIUTI, Valerio. Impugnazioni e principio di immediatezza nella giurisprudenza della Corte di Strasburgo. Diritto penale e processo, p. 1440-1451, 2015; AIUTI, Valerio. L'art. 603 c.p.p. dopo Dan c. Moldavia: un casebook. Giurisprudenza italiana, p. 1002-1019, 2016.

16 Posto che la regola non ha un valore assoluto, ma è suscettibile di essere derogata laddove sia impossibile risentire il testimone, come nel caso del suo decesso. In questo senso, Corte edu, Gr. Cam., 15 dicembre 2011, Al-Khawaja e Tahery c. Regno Unito, ricorsi n. 26766/05 e 22228/06, in https://hudoc. echr.coe.int/, su cui si veda BALSAMO, Antonio. La Corte di Strasburgo e i testimoni assenti: gli sviluppi del "nuovo corso" avviato dalla sentenza AlKhawaja. Cassazione penale, p. 2837-2847, 2013.

17 Testualmente Corte edu, I sez., 29 giugno 2017, Lorefice c. Italia, ricorso n. $63446 / 13, \S 43$. Per un commento alla decisione, BIONDI, Giuseppe. Tanto tuonò che piovve! La prima condanna dell'Italia da parte della Corte EDU in tema di overturning sfavorevole in appello: una sentenza (quasi) annunciata. Cassazione penale, p. 4556-4577, 2017; AIUTI, Valerio. Corte europea e "motivazione rafforzata" nel caso Lorefice. Cassazione penale, p. 682-690, 2018.

18 Tanto è vero che, prima che fosse intrapreso questo percorso di ripensamento, la giurisprudenza richiedeva, ai fini della riforma della sentenza di proscioglimento, la redazione di una mera motivazione "rafforzata", volta a confutare i più rilevanti argomenti della decisione gravata. In questo senso, Cass., sez. un., 12 luglio 2005, n. 33748, in Cassazione penale, p. 3732. 
condanna dell'imputato, del "ragionevole dubbio", alla luce del quale il Giudice di legittimità ha modellato i confini dell'obbligatorietà della rinnovazione istruttoria ai fini della riforma della sentenza di primo grado.

Con la prima decisione, è stato affermato come, allorché il pubblico ministero si dolga dinanzi alla corte d'appello dell'esito assolutorio di primo grado facendo valere l'erronea valutazione di una prova dichiarativa ${ }^{19}$, il canone Bard, ai fini della condanna, richieda di «replic[are] l'andamento del giudizio di primo grado, fondandosi su prove dichiarative direttamente assunte». Ne deriva l'obbligo di disporre la rinnovazione istruttoria, anche d'ufficio ai sensi del terzo comma dell'art. 603, in quanto soltanto il contatto diretto tra il giudice di seconda istanza e la fonte di prova può consentire di fugare ogni dubbio in ordine alla responsabilità dell'imputato e, quindi, di sovvertire il risultato liberatorio del primo giudizio ${ }^{20}$.

Alla medesima conclusione è pervenuta anche la seconda sentenza delle Sezioni Unite che, in tema di ribaltamento dell'assoluzione emessa al termine di un processo celebrato secondo le forme del rito abbreviato, anche non condizionato all'integrazione probatoria, hanno specificato come, ai fini dell'overturning, l'art. 533 c.p.p. imponga comunque il rispetto del principio di immediatezza in sede di appello, malgrado l'accertamento da parte del primo giudice si sia basato sulle "carte" contenute nel fascicolo del p.m. ${ }^{21}$.

Infine, la terza decisione ha escluso l'obbligo di riassumere la prova dichiarativa per convertire la condanna in assoluzione, atteso che

19 Categoria nella quale devono essere ricomprese la perizia e la consulenza tecnica (Cass., sez. un., 28 gennaio 2019, n. 14426, in Diritto penale e Processo, p. 822, 2019, con nota di BONZANO, Carlo. Le Sezioni Unite Pavan e la morte di un dogma: il contraddittorio per la prova spazza via la neutralità della perizia.

20 Cass., sez. un., 28 aprile 2016, n. 27620, disponibile in: <https://archiviodpc. dirittopenaleuomo.org/d/4953-reformatio-in-peius-in-appello-e-processo-equo-art-6-cedu-fisiologia-e-patologia-secondo-le-sezioni>. commentata da LORENZETTO, Elisa. Reformatio in peius in appello e processo equo (art. 6 CEDU): fisiologia e patologia secondo le Sezioni Unite.

21 Cass., sez. un., 19 gennaio 2017, n. 18620, in Cassazione penale, p. 2666, 2017, annotata da APRATI, Roberta. Overturning sfavorevole in appello e mancanza del riesame. Per un'analisi del quadro risultante da queste due pronunce, BELLUTA, Hervé, LUPÁRIA, Luca. La parabola ascendente dell'istruttoria in appello nell'esegesi "formante" delle Sezioni Unite. Diritto penale contemporaneo, Rivista trimestrale, v. 3, p. 151-162, 2017. 
l'incertezza in ordine alla colpevolezza dell'imputato ben può emergere anche dalla mera rilettura dei verbali di prove redatti nell'ambito del primo grado di giudizio ${ }^{22}$.

Le conclusioni cui si è giunti con questo trittico di decisioni sono in parte meritevoli di apprezzamento, posto che, in un'ottica di chiaro favor per l'imputato, si è tentato di interpretare l'art. 603 c.p.p. in modo conforme alla giurisprudenza convenzionale ${ }^{23}$. Tuttavia, risolta tale questione, ne è sorta un'altra, altrettanto spinosa, originata dalla combinazione tra l'impostazione inquisitoria del secondo grado di giudizio e il nuovo canale di ammissione delle prove dichiarative aperto a livello pretorio.

Infatti, viene da chiedersi quale sia l'ampiezza dell'obbligo della corte d'appello di rinnovare l'istruzione dibattimentale.

Con la sentenza del 2016, le Sezioni Unite hanno subordinato questo dovere alla circostanza che le dichiarazioni dei testimoni, sulla cui base procedere alla conversione della decisione impugnata, siano «ritenute decisive ai fini del giudizio assolutorio di primo grado» ${ }^{24}$, alla stessa stregua, del resto, dell'impostazione seguita dalla Corte europea ${ }^{25}$.

Sennonché, la dottrina ha condivisibilmente evidenziato i rischi sottesi ad una valutazione del giudice in ordine al peso rivestito dalla prova nell'economia della vicenda concreta: la verifica circa il carattere determinante dell'apporto di una fonte dichiarativa potrebbe «ritorcersi contro [...] l'imparzialità» del decidente, «lasciando presagire la propria

22 Cass., sez. un., 21 dicembre 2017, n. 14800, disponibile in: <https://archiviodpc.dirittopenaleuomo.org/d/5977-la-riassunzione-della-prova-dichiarativa-in-appello-note-a-margine-di-sezioni-unite-troise>, con nota di GALANTINI, Novella. La Riassunzione della prova dichiarativa in appello: note a margine di Sezioni Unite Troise.

23 Dà una valutazione complessivamente positiva dell'illustrato percorso giurisprudenziale, LAVARINI, Barbara. Sistema delle impugnazioni e profili di garanzia. Il passato e il presente. In: Aa.Vv. "Processo e garanzie della persona" - Atti del Convegno in onore di Mario Chiavario. Legislazione penale, p. 140, 19 ottobre 2020.

24 Cass., sez. un., 28 aprile 2016, n. 27620, cit.

25 Secondo la Corte edu, sono decisive le «testimonianze che avevano portato il tribunale a dubitare della fondatezza dell'accusa formulata nei suoi confronti a tal punto da motivare l'assoluzione di quest'ultimo» (Corte edu, III sez., 5 marzo 2013, Manolachi c. Romania, ricorso n. 36605/04, § 48). 
intenzione di condannare il prosciolto ogni qual volta si determini per la rinnovazione della prova dichiarativa ${ }^{26}$. Senza contare come un simile vaglio mal si attaglia ad un controllo preliminare come quello da operare in sede di ammissione delle prove, visto che solo dopo l'assunzione delle stesse può essere svolto con la dovuta consapevolezza ${ }^{27}$.

Così, il legislatore, nell'introdurre l'art. 603 comma 3-bis, ha scelto di non includere tale requisito nella trama della disposizione. Ma anche questa soluzione, come vedremo, è suscettibile di dare adito ad una serie di dubbi.

\section{L'ART. 603 COMMA 3-BIS TRA DIRITTO DI DIFESA E PARITÀ DELLE PARTI}

La 1. 23 giugno 2017, n. 103 ("riforma Orlando"), ha inserito nel tessuto dell'art. 603 c.p.p. il comma 3-bis, a mente del quale «nel caso di appello del pubblico ministero contro una sentenza di proscioglimento per motivi attinenti alla valutazione della prova dichiarativa, il giudice dispone la rinnovazione dell'istruzione dibattimentale».

Già da una prima lettura della disposizione emergono due importanti differenze tra le impostazioni seguite dal legislatore e dalla giurisprudenza: da un lato, il presupposto cui è subordinato l'obbligo di rinnovazione è che l'accusa, nell'atto di impugnazione proposta contro una pronuncia liberatoria, si dolga della valutazione del primo giudice in ordine alle dichiarazioni di una fonte di prova; dall'altro, la riassunzione

26 In questi termini, BELLUTA, Hervé. LUPÁRIA, Luca. La rinnovazione dell'istruzione dibattimentale tra legge e giurisprudenza: punti fermi ... e non. In: CANZIO, Giovanni, BRICCHETTI, Renato. Le impugnazioni penali. Milano: Giuffrè, 2019, p. 358. Anche CAPONE, Arturo. Appello del pubblico ministero e rinnovazione istruttoria. In: BARGIS, Marta, BELLUTA, Hervé (a cura di). La riforma delle impugnazioni tra carenze sistematiche e incertezze applicative (commento alla legge 23 giugno 2017, n. 103 e al d.lgs. 6 febbraio 2018, n. 11). Torino: Giappichelli, 2018, p. 67, evidenzia alcune controindicazioni cui avrebbe potuto dar luogo il parametro della "decisività".

In questo senso, CHINNICI, Daniela. Contraddittorio e giudizio di appello. Ortodossia europea, resistenze interne e graduali aperture in attesa del "sigillo" del legislatore. In: NEGRI, Daniele, ORLANDI, Renzo (a cura di). Le erosioni silenziose del contraddittorio. Torino: Giappichelli, 2017, p. 210. 
della prova è «automatic[a] $\gg^{28}$, cioè non sottoponibile ad alcuna indagine in punto di ammissibilità da parte della corte d'appello che, una volta verificata, ai sensi dell'art. 581 c.p.p., la specificità del motivo con cui il p.m. contesta il modo in cui è stata considerata la testimonianza ${ }^{29}$, è tenuta a disporre il supplemento istruttorio, a prescindere da un'esplicita richiesta in tal senso ${ }^{30}$.

La ratio della prescrizione normativa si pone nello stesso solco garantista della giurisprudenza europea e interna, con l'obiettivo di assicurare il rispetto dei principi del contraddittorio, dell'oralità e dell'immediatezza tutte le volte in cui vi possano essere i margini per la riforma di una sentenza liberatoria.

Tuttavia, il legislatore ha avuto il demerito di considerare la riassunzione della prova dichiarativa in appello soltanto come strumento di tutela dell'imputato, al quale si consente di partecipare nuovamente all'esame incrociato dinanzi al giudice chiamato a rivalutare la sua responsabilità, ignorando colpevolmente le potenzialità persuasive offerte dal metodo dialettico di formazione della prova. In altre parole, poiché l'obbligo di riassunzione della fonte dichiarativa è previsto unicamente in relazione all'appello esperito dall'accusa, quest'ultima è stata dotata di uno strumento estremamente efficace per perorare la propria causa, in assenza di adeguate contromisure in favore dell'imputato ${ }^{31}$.

28 Utilizza questa espressione, CANESCHI, Gaia. La rinnovazione istruttoria in appello dopo la riforma Orlando: una non soluzione ad un problema apparente. Rivista italiana di diritto e procedura penale, p. 841, 2018.

29 Ritiene che il vaglio sulla specificità dei motivi di appello coinvolga anche questo profilo, CAPONE, Arturo. Appello del pubblico ministero e rinnovazione istruttoria. In: BARGIS, Marta, BELLUTA, Hervé (a cura di). La riforma delle impugnazioni tra carenze sistematiche e incertezze applicative (commento alla legge 23 giugno 2017, n. 103 e al d.lgs. 6 febbraio 2018, n. 11). Torino: Giappichelli, 2018, p. 69.

30 Di qui i dubbi circa il rispetto del principio dell'imparzialità del giudice, il quale si «converte $[. .$.$] in investigatore»: la disposizione «favorisce l'uso del$ potere istruttorio in funzione della verifica di una (ormai divenuta propria) ipotesi ricostruttiva del fatto» (MARANDOLA, Antonella. L'appello riformato. Milano: Cedam, 2020, p. 188)

31 Come osservato da CAPONE, Arturo. Prova in appello: un difficile bilanciamento. Processo penale e giustizia, v. 6, p. 52, 2016, «la critica dell'assoluzione si avvale di regola di una nuova escussione incrociata, in cui il pubblico 
Difatti, al fine di ottenere la riammissione di altre prove, diverse da quelle su cui si sono concentrati i motivi d'appello del p.m., la difesa deve passare dalle "strettoie" del primo comma e del terzo comma dell'art. 603. D’altronde, benché il nuovo comma 3-bis si esprima in termini di «rinnovazione dell'istruttoria dibattimentale», le Sezioni Unite hanno specificato come questa formula, lungi dall'aprire le porte ad una riedizione dell'attività istruttoria già svolta in primo grado, non imponga di assumere nuovamente tutte le prove, ben potendo essere escussa la sola «fonte la cui dichiarazione sia oggetto di una specifica censura da parte del pubblico ministero attraverso la richiesta di una nuova valutazione da parte del giudice d'appello». Per il resto - prosegue il Giudice di legittimità - un eventuale ulteriore segmento istruttorio potrà essere disposto dal giudice qualora lo ritenga assolutamente necessario ex art. 603 comma 3 c.p.p. ${ }^{32}$.

L'art. 603 comma 3-bis, quindi, è suscettibile di dar luogo ad un'indebita disparità di trattamento tra il pubblico ministero, dei cui poteri peraltro gode anche la parte civile, e l'imputato.

È piuttosto singolare rilevare come sia la riforma del 2006, sia quella del 2017, pur perseguendo un obiettivo comune - impedire il ribaltamento in appello della sentenza di proscioglimento sulla base degli atti - con strumenti sensibilmente diversi, finiscano per destare i medesimi dubbi sul piano della parità delle parti. Ma se il soggetto che usciva "penalizzato" dopo l'entrata in vigore del primo dei due citati interventi legislativi era il pubblico ministero, la legge Orlando ha dato luogo ad un più grave squilibrio a sfavore dell'imputato, la cui posizione, in sede di impugnazione, dovrebbe invece essere oggetto di una disciplina (quantomeno) analoga a quella dell'accusa, posto che è in gioco la sua libertà personale.

Da questo punto di vista, riecheggiano le critiche mosse dalla dottrina nei confronti della già richiamata sentenza del 2007 del Giudice delle leggi che, proprio in nome del principio di cui all'art. 111, comma 2, Cost., aveva dichiarato l'illegittimità del divieto per la procura di

ministero potrà tentare di mettere in luce le incoerenze dei testimoni considerati attendibili in primo grado. Non così accade nel caso inverso».

32 Cass., sez. un., 21 dicembre 2017, n. 14800, in Rivista italiana di diritto e procedura penale, p. 274, 2019. 
appellare le sentenze di proscioglimento ${ }^{33}$. I commentatori, in particolare, imputarono alla Corte di non aver individuato una base costituzionale, diversa dall'equality of arms, cui ancorare il potere dell'accusa di proporre appello, la rilevanza del quale era stata esclusivamente legata al fatto che all'imputato fosse «consentito l'esercizio di un omologo diritto» ${ }^{34}$. In altre parole, ai giudici della Consulta si contestò di aver omesso di considerare la natura anzitutto difensiva del mezzo di impugnazione, che ha la principale funzione di garantire al condannato un controllo sulla decisione di primo grado ${ }^{35}$.

In quest'ottica, deve essere salutata con favore una recente pronuncia della stessa Corte costituzionale che, richiesta di valutare la compressione - operata dal d.lgs. 6 febbraio 2018, n. 11 - della legittimazione del p.m. di appellare le sentenze di condanna, ne ha affermato la piena legittimità, rilevando come la mancanza di «una contrapposta limitazione, di analogo spessore, dal lato dell'imputato, rientra nella logica della diversa quotazione costituzionale del potere di impugnazione delle due parti necessarie del processo penale: privo di autonoma copertura nell'art. 112 Cost. - e, dunque, più "malleabile", in funzione della realizzazione di interessi contrapposti - quello della parte pubblica; intimamente collegato, invece, all'art. 24 Cost. - e, dunque, meno disponibile a interventi limitativi - quello dell'imputato» ${ }^{36}$.

Ebbene, il principio dell'inviolabilità del diritto di difesa non può tollerare un così evidente divario tra gli spazi concessi dall'art. 603

33 Corte cost., 24 gennaio 2007 (dep. 6 febbraio 2007), n. 26, in Giurisprudenza costituzionale, p. 221, 2007.

34 In questi termini DE CARO, Agostino. Inappellabilità delle sentenze di assoluzione da parte del p.m.: l'incostituzionalità della "Pecorella". Diritto penale e processo, p. 610, 2007. Nello stesso senso MARZADURI, Enrico. Sistema da riscrivere dopo ampie riflessioni. Guida al diritto, v. 8, p. 85, 2007.

35 In tal senso FILIPPI, Leonardo. La Corte costituzionale disegna un processo accusatorio “all'italiana”. In FILIPPI, Leonardo (a cura di). Il nuovo regime delle impugnazioni tra Corte costituzionale e Sezioni Unite. Padova: Cedam, 2007, p. 3 ss.

36 Corte cost., 4 dicembre 2019 (dep. 4 marzo 2020), n. 34, disponibile in: <https:// www.sistemapenale.it/it/sentenza/santalucia-nota-corte-cost-34-2020-appello-riforma-orlando>., con nota di SANTALUCIA, Giuseppe, Il futuro dell'appello nelle ragioni di compatibilità costituzionale della riforma Orlando. 
comma 3-bis al pubblico ministero per ottenere la rinnovazione istruttoria e quelli, decisamente meno ampi, a disposizione dell'imputato.

Il legislatore, sebbene intervenuto in un'ottica di garanzia, ha infatti fornito all'accusatore «un'arma» contro le sentenze di proscioglimento, capace di esporre l'accusato «alla particolare forza di convincimento tipica del contatto diretto tra il giudice e il dichiarante, contrapponendovi soltanto la lettura (magari soltanto potenziale) di precedenti deposizioni trasfuse nelle carte processuali» ${ }^{37}$. Ciò dovrebbe implicare il diritto della difesa di chiedere e ottenere, ai sensi dell'art. 495 comma 2 c.p.p., l'ammissione della prova contraria ${ }^{38}$, facoltà che invece è esclusa dall'attuale conformazione dell'art. 603, così come interpretato dalla Corte di cassazione.

\section{L'AMMISSIONE DELLE PROVE DICHIARATIVE IN CASO DI APPELLO DELL'IMPUTATO AVVERSO LA SENTENZA DI CONDANNA}

Oltre a pregiudicare la posizione dell'imputato nei giudizi di appello promossi nei confronti di decisioni assolutorie, l'art. 603 comma 3-bis, nel riferirsi all'«appello del pubblico ministero contro una sentenza di proscioglimento», esclude dal proprio perimetro di applicazione le ipotesi di impugnazione promossa dall'imputato avverso una sentenza di condanna, come confermato dalle Sezioni Unite con l'ultima delle tre pronunce sopra ricordate ${ }^{39}$.

Ne deriva un quadro profondamente problematico, in cui la difesa viene a trovarsi in una posizione deteriore rispetto alla controparte non soltanto nel processo in cui si discuta della riforma del proscioglimento, ma anche della condanna, poiché la normativa di nuovo conio determina

37 Testualmente BALSAMO, Antonio. La rinnovazione dell'istruttoria dibattimentale. In: PULVIRENTI, Antonino (a cura di). Le impugnazioni penali dopo la riforma. Torino: Giappichelli, 2018, p. 179.

38 Ibidem, p. 180. Ad avviso dell'Autore, se questo diritto fosse riconosciuto, «il processo di appello potrebbe così iniziare una vera e propria mutazione genetica, capace di migliorare la qualità della decisione giudiziaria, di rafforzare la motivazione personale dei suoi protagonisti, di seguire una impostazione pienamente coerente con i parametri europei».

39 Cass., sez. un., 21 dicembre 2017, n. 14800, in Rivista italiana di diritto e procedura penale, p. 274, 2019. 
un sistematico mismatch tra le chances dei due soggetti necessari del processo di ottenere la modifica della sentenza che li vede - per utilizzare una pur criticata ma efficace espressione della Corte costituzionale rispettivamente "soccombenti".

La dottrina ha sin da subito colto questo "effetto collaterale" della riforma, che attribuisce alla procura un vero e proprio «diritto potestativo ${ }^{40}$ alla riassunzione delle prove orali erroneamente valutate in primo grado, di modo che la corte d'appello possa avere un contatto diretto con il dichiarante, del quale deve saggiare la credibilità. Al contrario, l'imputato rimane dotato della esclusiva facoltà di sollecitare i giudici a utilizzare i poteri istruttori conferiti loro dall'art. $603^{41}$. In altri termini, ci si chiede per quale ragione il condannato non possa beneficiare dello stesso strumentario a disposizione dell'accusa ai fini della riforma delle pronunce di proscioglimento.

Questa controindicazione della prescrizione introdotta nel 2017 rischia di rendere vani gli sforzi del nostro ordinamento per adeguarsi ai dicta della Corte di Strasburgo, il cui compito è esattamente quello di assicurare che ciascuna parte abbia avuto una ragionevole possibilità di far valere la propria posizione in condizioni tali da non porla in una situazione di netto svantaggio rispetto "all'avversario"42.

40 Impiega questo termine CAPONE, Arturo. Appello del pubblico ministero e rinnovazione istruttoria. In: BARGIS, Marta, BELLUTA, Hervé (a cura di). La riforma delle impugnazioni tra carenze sistematiche e incertezze applicative (commento alla legge 23 giugno 2017, n. 103 e al d.lgs. 6 febbraio 2018, n. 11). Torino: Giappichelli, 2018, p. 68.

41 Ibidem, p. 77. Nello stesso senso CERESA-GASTALDO, Massimo. La riforma dell'appello, tra malinteso garantismo e spinte deflative. Diritto penale contemporaneo, Rivista trimestrale, v. 3, p. 168, 2017, e NACAR, Barbara. La rinnovazione dell'istruttoria dibattimentale in appello: dubbi applicativi e questioni di legittimità costituzionale. Diritto penale e processo, p. 314 ss., 2018. Contra BRONZO, Pasquale. La nuova ipotesi di rinnovazione dell'istruttoria dibattimentale in appello. In: BACCARI, Gian Marco, BONZANO, Carlo, LA REGINA, Katia, MANCUSO, Enrico Maria (a cura di). Le recenti riforme in materia penale. Dai decreti di depenalizzazione (d.lgs. n. 7 e n. 8/2016) alla legge "Orlando" (l. n. 103/2017). Padova: Cedam, 2017, p. 417, ad avviso del quale, anzi la disposizione condiziona l'accoglimento delle critiche del p.m., anche laddove essere fossero del tutto persuasive, all'espletamento di attività istruttoria.

42 Cfr. Corte edu, II sez., 20 dicembre 2005, Guillemot c. Francia, ricorso n. 21922/03, § 48, in https://hudoc.echr.coe.int/. Il Giudice europeo, peraltro, 
La Corte di cassazione è conscia del pericolo che si nasconde dietro l'art. 603 comma 3-bis, tanto è vero che si sforza di circoscriverne la portata: richiesta dal procuratore generale se la previsione debba essere interpretata nel senso che la corte di appello, investita del gravame avverso una sentenza di assoluzione della parte pubblica fondato sulla valutazione di prove dichiarative, sia o meno obbligata a rinnovare l'istruttoria, ha dato una risposta negativa: il giudice di seconda istanza è tenuto «a sindacare se i motivi stessi siano ammissibili, perché formulati in ossequio ai criteri indicati dall'art. 581 c.p.p., e se le prove indicate siano decisive». Inoltre, la decisione sulla riassunzione della prova non deve necessariamente essere presa «in limine litis ma anche all'esito della discussione e consentito, comunque, il contraddittorio delle parti» ${ }^{43}$.

Questa consapevolezza, tuttavia, non esclude il verificarsi di situazioni patologiche, in cui l'imputato venga a trovarsi in una posizione di subalternità rispetto al p.m., cui ben può dar luogo l'infelice formulazione della disposizione esaminata.

\section{Considerazioni conclusive}

Nei paragrafi precedenti si è tentato di dimostrare come l'innesto di una prescrizione normativa, volta a garantire la formazione della prova nel contraddittorio tra le parti in vista del ribaltamento della sentenza di proscioglimento, nel tessuto di un appello a trazione chiaramente inquisitoria, sia suscettibile di incidere negativamente sull'equilibrio generale del processo.

Serve allora un cambio di rotta, nel senso di rendere il giudizio di seconda istanza maggiormente conforme ai principi costituzionali in

non ha mancato di rilevare una violazione dell'art. 6 CEDU in un'ipotesi in cui il giudice dell'impugnazione, dinanzi al gravame interposto avverso una sentenza di condanna, ha riqualificato in peius il fatto, aumentando la pena detentiva precedentemente irrogata, senza disporre la nuova audizione dei dichiaranti (Corte edu, IV sez., 4 ottobre 2016, ricorso n. 27018/06, Torja c. Romania, in https://hudoc.echr.coe.int/. Per un'analisi della decisione si veda TESORIERO, Salvatore. Riforma della sentenza e riapertura dell'istruttoria in appello. Archivio penale Web, v. 2, p. 1-26, 2017).

43 Si veda Cass., sez. V, 16 aprile 2019, n. 19730, in Cassazione penale, p. $1612,2020$. 
materia di giusto processo e, soprattutto, di farne uno strumento che abbia primariamente la funzione di tutelare l'imputato, a maggior ragione laddove ne sia stata dichiarata la colpevolezza in primo grado.

Del resto, com'è stato autorevolmente osservato, dalla giurisprudenza della Corte europea dei diritti dell'uomo emerge «that appeal represents an instrument prevailingly in favour of the defence», cui viene attribuita «a further chance [...] to overturn the negative outcome of the trial (or to mitigate its effects) ${ }^{44}$. Senza contare che, da un confronto tra le principali fonti sovranazionali in materia - segnatamente l'art. 2 del Protocollo n. 7 allegato alla CEDU, l'art. 14 par. 5 PIDCP, nonché, per quanto interessa l'ordinamento brasiliano, l'art. 8 par. 2 lett. $h \mathrm{CADU}^{45}-$, si ricava chiaramente la maggiore attenzione da dedicare, nel campo delle impugnazioni, al controllo circa la correttezza delle decisioni di condanna.

Sotto questo profilo, prendendo spunto dalla double jeopardy clause statunitense - che impedisce all'accusa di impugnare la sentenza di proscioglimento -, la dottrina brasiliana non ha mancato di criticare il paradigma della bilateralità dei mezzi di impugnazione, sul presupposto, per un verso, che «o direito ao recurso, como norma fundamental, tutela unicamente o imputado» raggiunto da una sentenza di condanna e, per l'altro, che il principio del ne bis in idem impedisce lo svolgimento «de um novo julgamento de mérito após uma sentença absolutória» ${ }^{46}$.

In effetti, al netto delle resistenze della Corte costituzionale, l'idea di "ristrutturare" l'appello in chiave prettamente difensiva pare essere convenzionalmente plausibile: una riforma dell'istituto orientata a salvaguardare la libertà dell'imputato - condannato o raggiunto da

44 Così CAIANIELLO, Michele. Criminal appeals in Europe. The prospective of the defence. European Journal of Crime, Criminal Law and Criminal Justice, v. 24, n. 4, p. 288, 2016.

Sul valore di questa Convenzione nella gerarchia brasiliana delle fonti del diritto, RUGGERI, Stefano. La riforma delle impugnazioni al vaglio del diritto comparato. In: BARGIS, Marta, BELLUTA, Hervé (a cura di). La riforma delle impugnazioni tra carenze sistematiche e incertezze applicative (commento alla legge 23 giugno 2017, n. 103 e al d.lgs. 6 febbraio 2018, n. 11). Torino: Giappichelli, 2018, p. 280 ss.

46 Per queste considerazioni v. GLOECKNER, Ricardo Jacobsen. Direito fundamental ao recurso no processo penal: uma crítica à concepção bilateral da impugnação. Revista Justiça e Sistema Criminal, v. 6, n. 11, p. 193 e 200, 2014. 
una sentenza di proscioglimento non pienamente liberatoria -, con facoltà di ottenere la riassunzione delle prove che si ritengono essere state erroneamente valutate dal primo giudice, garantirebbe il rispetto dei principi espressi dall'art. 111 Cost. anche in sede di seconda istanza. Una siffatta apertura, di per sé idonea rappresentare un pericolo per la tenuta complessiva del sistema, necessiterebbe di un contrappeso, individuabile nell'introduzione di un "filtro" in entrata per impedire la trattazione di gravami meramente pretestuosi e finalizzati a ritardare l'esecuzione della sentenza ${ }^{47}$.

Sfortunatamente, il legislatore italiano non appare ancora pronto ad intraprendere una riflessione tesa al ripensamento del rimedio in esame, preferendo continuare a muoversi lungo la strada tracciata nel 1988. Anzi, in un'ottica di efficienza, si sta sempre più orientando nel senso di un ulteriore impoverimento delle garanzie, con un'«erosione progressiva di spazi di effettività alla piena realizzazione del controllo di merito» ${ }^{48}$.

Difatti, le più recenti novità sono rappresentate, in primo luogo, dal disegno di legge di riforma della giustizia penale C. 2435, approdato alla Camera lo scorso marzo, che in materia di appello prevede, quale principale modifica, l'introduzione della regola del giudice monocratico per un ampio novero di fattispecie criminose ${ }^{49}$; in secondo luogo, dal d.l. 9 novembre 2020, n. 149 (c.d. "Ristori bis") - emanato nell'ambito dei provvedimenti tesi ad affrontare la crisi pandemica -, il cui art. 23 prevede che fino al 31 gennaio 2021 i processi di secondo grado si svolgeranno, di

47 Da ultimo, ha evidenziato l'opportunità di istituire un meccanismo di selezione degli atti di appello CANZIO, Giovanni. Le impugnazioni penali tra riforme legislative e diritto giurisprudenziale. In: CANZIO, Giovanni, BRICCHETTI, Renato. Le impugnazioni penali. Milano: Giuffrè, 2019, p. 18 s.

48 Si esprime in questi termini DE CARO, Agostino. Riflessioni sulla legittimità costituzionale dell'appello alla vigilia del dibattito parlamentare sulla riforma di parte del processo penale. Archivio penale Web, v. 2, p. 18, 2020.

49 Per un commento alle proposte del Governo si vedano, tra gli altri, GIALUZ, Mitja, DELLA TORRE, Jacopo. Il progetto governativo di riforma della giustizia penale approda alla Camera: per avere processi rapidi (e giusti) serve un cambio di passo. Sistema penale, v. 4, p. 145-201, 2020. Sullo specifico tema della monocraticità in appello, volendo, AGOSTINO, Lorenzo. Il giudizio d'appello monocratico che verrà. Disponibile in: http://www.lalegislazionepenale.eu/wp-content/uploads/2020/07/Agostino-Approfondimenti.pdf>. 
regola, senza la partecipazione delle parti ${ }^{50}$. Tali scelte, benché assunte in un periodo emergenziale, «non lasciano certamente intravedere un futuro luminoso per il giudizio di appello», ma «anzi rivelano la tendenza ad una sua trasfigurazione, come se il controllo di merito fosse un ostacolo nel percorso verso la celere formazione della res iudicata ${ }^{51}$.

Manca, in definitiva, una visione sistematica, capace di ovviare a problemi avvertiti ormai da troppo tempo ${ }^{52}$.

\section{Bibliografia}

AGOSTINO, Lorenzo. Il giudizio d'appello monocratico che verrà. Disponibile in: http://www.lalegislazionepenale.eu/wp-content/uploads/2020/07/Agostino-Approfondimenti.pdf >.

AIUTI, Valerio. Impugnazioni e principio di immediatezza nella giurisprudenza della Corte di Strasburgo. Diritto penale e processo, p. 1440-1451, 2015.

AIUTI, Valerio. L'art. 603 c.p.p. dopo Dan c. Moldavia: un casebook. Giurisprudenza italiana, p. 1002-1019, 2016.

AIUTI, Valerio. Corte europea e "motivazione rafforzata" nel caso Lorefice. Cassazione penale, p. 682-690, 2018.

APRATI, Roberta. Overturning sfavorevole in appello e mancanza del riesame. Cassazione penale, p. 2672-2682, 2017.

BALSAMO, Antonio. La Corte di Strasburgo e i testimoni assenti: gli sviluppi del "nuovo corso" avviato dalla sentenza Al-Khawaja. Cassazione penale, p. 28372847, 2013.

50 Per un primo commento al decreto, MARANDOLA, Antonella. Decreto Ristori bis: l'appello cartolare (temporaneo?) e l'allungamento della prescrizione e dei termini di custodia cautelare nel periodo di emergenza. Disponibile in: <https://ilpenalista.it/articoli/news/decreto-ristori-bis-lappello-cartolare-temporaneo-e-l-allungamento-della-prescrizione>.

51 Per queste considerazioni MANGIARACINA, Annalisa. Prove tecniche per la "soppressione" del giudizio di appello? Archivio penale Web, v. 3, p. 1, 2020.

52 Si pongono in controtendenza rispetto agli ultimi interventi normativi le modifiche al sistema delle impugnazioni contenute all'interno della proposta di riforma della giustizia penale recentemente elaborata dalla Commissione Lattanzi, consultabile all'indirizzo https://www.gnewsonline.it/ ddl-penale-ecco-la-relazione-finale-della-commissione-lattanzi/. 
BALSAMO, Antonio. La rinnovazione dell'istruttoria dibattimentale. In: PULVIRENTI, Antonino (a cura di). Le impugnazioni penali dopo la riforma. Torino: Giappichelli, 2018, p. 167.

BARGI, Alfredo. I nuovi ambiti oggettivi delle impugnazioni. In: GAITO, Alfredo (a cura di). La nuova disciplina delle impugnazioni dopo la "legge Pecorella". Torino: Utet, 2006, p. 151.

BELLUTA, Hervé. Prospettive di riforma dell'appello penale: tra modifiche strutturali e microchirurgia normativa. In: BARGIS, Marta, BELLUTA, Hervé. Impugnazioni penali. Assestamenti del sistema e prospettive di riforma. Torino: Giappichelli, 2013, p. 235.

BELLUTA, Hervé, LUPÁRIA, Luca. La parabola ascendente dell'istruttoria in appello nell'esegesi “formante" delle Sezioni Unite. Diritto penale contemporaneo, Rivista trimestrale, v. 3, p. 151-162, 2017.

BELLUTA, Hervé, LUPÁRIA, Luca. La rinnovazione dell'istruzione dibattimentale tra legge e giurisprudenza: punti fermi ... e non. In: CANZIO, Giovanni, BRICCHETTI, Renato. Le impugnazioni penali. Milano: Giuffrè, 2019, p. 345.

BIONDI, Giuseppe. Tanto tuonò che piovve! La prima condanna dell'Italia da parte della Corte EDU in tema di overturning sfavorevole in appello: una sentenza (quasi) annunciata. Cassazione penale, p. 4556-4577, 2017.

BONZANO, Carlo. Le Sezioni Unite Pavan e la morte di un dogma: il contraddittorio per la prova spazza via la neutralità della perizia. Diritto penale e processo, p. 822-831, 2019.

BRONZO, Pasquale. La nuova ipotesi di rinnovazione dell'istruttoria dibattimentale in appello. In: BACCARI, Gian Marco, BONZANO, Carlo, LA REGINA, Katia, MANCUSO, Enrico Maria (a cura di). Le recenti riforme in materia penale. Dai decreti di depenalizzazione (d.lgs. n. 7 e n. 8/2016) alla legge "Orlando" (1. n. 103/2017). Padova: Cedam, 2017, p. 409.

CAIANIELLO, Michele. Criminal appeals in Europe. The prospective of the defence. European Journal of Crime, Criminal Law and Criminal Justice, v. 24, n. 4, p. 274-290, 2016.

CANESCHI, Gaia. La rinnovazione istruttoria in appello dopo la riforma Orlando: una non soluzione ad un problema apparente. Rivista italiana di diritto e procedura penale, p. 821-857, 2018.

CANZIO, Giovanni. Le impugnazioni penali tra riforme legislative e diritto giurisprudenziale. In: CANZIO, Giovanni, BRICCHETTI, Renato. Le impugnazioni penali. Milano: Giuffrè, 2019, p. 1. 
CAPONE, Arturo. Prova in appello: un difficile bilanciamento. Processo penale $e$ giustizia, v. 6, p. 52-65, 2016.

CAPONE, Arturo. Appello del pubblico ministero e rinnovazione istruttoria. In: BARGIS, Marta, BELLUTA, Hervé (a cura di). La riforma delle impugnazioni tra carenze sistematiche e incertezze applicative (commento alla legge 23 giugno 2017, n. 103 e al d.lgs. 6 febbraio 2018, n. 11). Torino: Giappichelli, 2018, p. 53.

CAPRIOLI, Francesco. Inappellabilità delle sentenze di proscioglimento e "parità delle armi” nel processo penale. Giurisprudenza costituzionale, p. 250-269, 2007.

CERESA-GASTALDO, Massimo. La riforma dell'appello, tra malinteso garantismo e spinte deflative. Diritto penale contemporaneo, Rivista trimestrale, v. 3, p. 163-172, 2017.

CHINNICI, Daniela. Giudizio penale di seconda istanza e giusto processo. Torino: Giappichelli, 2009.

CHINNICI, Daniela. Contraddittorio e giudizio di appello. Ortodossia europea, resistenze interne e graduali aperture in attesa del "sigillo" del legislatore. In: NEGRI, Daniele, ORLANDI, Renzo (a cura di). Le erosioni silenziose del contraddittorio. Torino: Giappichelli, 2017, p. 187.

DE CARO, Agostino. Inappellabilità delle sentenze di assoluzione da parte del p.m.: l'incostituzionalità della "Pecorella". Diritto penale e processo, p. 605-618, 2007.

DE CARO, Agostino. Riflessioni sulla legittimità costituzionale dell'appello alla vigilia del dibattito parlamentare sulla riforma di parte del processo penale. Archivio penale Web, v. 2, p. 1-19, 2020.

FILIPPI, Leonardo. La Corte costituzionale disegna un processo accusatorio “all'italiana”. In FILIPPI, Leonardo (a cura di). Il nuovo regime delle impugnazioni tra Corte costituzionale e Sezioni Unite. Padova: Cedam, 2007, p. 3.

GALANTINI, Novella. La Riassunzione della prova dichiarativa in appello: note a margine di Sezioni Unite Troise. Disponibile in: <https://archiviodpc.dirittopenaleuomo.org/d/5977-la-riassunzione-della-prova-dichiarativa-in-appello-note-a-margine-di-sezioni-unite-troise>.

GIACOMOLLI, Nereu José. Algumas marcas inquisitoriais do Código de Processo Penal brasileiro e a resistência às reformas. Revista Brasileira de Direito Processual Penal, vol. 1, n. 1, p. 143-165, 2015. https://doi.org/10.22197/rbdpp.v1i1.8

GIALUZ, Mitja, DELLA TORRE, Jacopo. Il progetto governativo di riforma della giustizia penale approda alla Camera: per avere processi rapidi (e giusti) serve un cambio di passo. Sistema penale, v. 4, p. 145-201, 2020. 
GLOECKNER, Ricardo Jacobsen. Direito fundamental ao recurso no processo penal: uma crítica à concepção bilateral da impugnação. Revista Justiça e Sistema Criminal, v. 6, n. 11, p. 183-203, 2014.

GRINOVER, Ada Pellegrini, MAGALHÃES, Antonio, SCARANCE, Antonio. Recursos no Processo Penal. 5a ed. São Paulo: RT, 2008.

LAVARINI, Barbara. Sistema delle impugnazioni e profili di garanzia. Il passato e il presente. In: Aa.Vv. "Processo e garanzie della persona" - Atti del Convegno in onore di Mario Chiavario. Legislazione penale, p. 134-140, 19 ottobre 2020.

LOPES JR., Aury. Direito processual penal. 17ª ed. São Paulo: Saraiva Educação, 2020.

LORENZETTO, Elisa. Reformatio in peius in appello e processo equo (art. 6 CEDU): fisiologia e patologia secondo le Sezioni Unite. Disponibile in: <https://archiviodpc. dirittopenaleuomo.org/d/4953-reformatio-in-peius-in-appello-e-processo-equoart-6-cedu-fisiologia-e-patologia-secondo-le-sezioni>.

LOZZI, Gilberto. Lezioni di procedura penale. 12a ed. Torino: Giappichelli, 2017. MANGIARACINA, Annalisa. Prove tecniche per la "soppressione" del giudizio di appello? Archivio penale Web, v. 3, p. 1-10, 2020.

MARANDOLA, Antonella. Nuovo regime dei casi d'appello. In: SCALFATI, Adolfo (a cura di). Novità su impugnazioni penali e regole di giudizio. Trento: Ipsoa, 2006, p. 121.

MARANDOLA, Antonella. Decreto Ristori bis: l'appello cartolare (temporaneo?) e l'allungamento della prescrizione e dei termini di custodia cautelare nel periodo di emergenza. Disponibile in: <https://ilpenalista.it/articoli/news/decreto-ristori-bis-lappello-cartolare-temporaneo-e-l-allungamento-della-prescrizione >

MARANDOLA, Antonella. L'appello riformato. Milano: Cedam, 2020.

MARZADURI, Enrico. Sistema da riscrivere dopo ampie riflessioni. Guida al diritto, v. 8, p. 85-86, 2007.

NACAR, Barbara. La rinnovazione dell'istruttoria dibattimentale in appello: dubbi applicativi e questioni di legittimità costituzionale. Diritto penale e processo, p. 314-331, 2018.

PRESUTTI, Adonella. L'inappellabilità delle sentenze di proscioglimento tra regola ed eccezione. In: BARGIS, Marta, CAPRIOLI, Francesco (a cura di). Impugnazioni e regole di giudizio nella legge di riforma del 2006. Dai problemi di fondo ai primi responsi costituzionali. Torino: Giappichelli, 2007, p. 51. 
RUGGERI, Stefano. La riforma delle impugnazioni al vaglio del diritto comparato. In: BARGIS, Marta, BELLUTA, Hervé (a cura di). La riforma delle impugnazioni tra carenze sistematiche e incertezze applicative (commento alla legge 23 giugno 2017, n. 103 e al d.lgs. 6 febbraio 2018, n. 11). Torino: Giappichelli, 2018, p. 271.

SANTALUCIA, Giuseppe. Il futuro dell'appello nelle ragioni di compatibilità costituzionale della riforma Orlando. Disponibile in: <https://www.sistemapenale. it/it/sentenza/santalucia-nota-corte-cost-34-2020-appello-riforma-orlando>.

TESORIERO, Salvatore. La rinnovazione della prova dichiarativa in appello alla luce della CEDU. Diritto penale contemporaneo, Rivista trimestrale, v. 3-4, p. 239276, 2014.

TESORIERO, Salvatore. Riforma della sentenza e riapertura dell'istruttoria in appello. Archivio penale Web, v. 2, p. 1-26, 2017.

ZAPPALÀ, Enzo. Sub art. 603. In: CHIAVARIO, Mario (coordinato da). Commento al nuovo codice di procedura penale, VI. Torino: Utet, 1991, p. 200.

\section{Additional information and author's declarations (scientific integrity)}

Conflict of interest declaration: the author confirms that there are no conflicts of interest in conducting this research and writing this article.

Declaration of authorship: all and only researchers who comply the authorship requirements of this article are listed as authors; all coauthors are fully responsible for this work in its entirety.

Declaration of originality: the author assures that the text here published has not been previously published in any other resource and that future republication will only take place with the express indication of the reference of this original publication; he also attests that there is no third party plagiarism or self-plagiarism. 
Editorial process dates

(http://www.ibraspp.com.br/revista/index.php/RBDPP/about/editorialPolicies)

- Submission: 02/12/2020

Editorial team

- Desk review and plagiarism check: $20 / 12 / 2020$

- Editor-in-chief: 1 (VGV)

- Review 1: 25/01/2021

- Associated-editor: 1 (BC)

- Review 2: 28/01/2021

- Reviewers: 2

- Transfer to V7N2: 23/02/2021

- Preliminary editorial decision: 16/05/2021

- Correction round return: 10/06/2021

- Final editorial decision: 11/06/2021

\section{HOW TO CITE (ABNT BRAZIL):}

AGOSTINO, Lorenzo. L'ammissione della prova in appello tra diritto di difesa e parità delle parti. Revista Brasileira de Direito Processual Penal, vol. 7, n. 2, p. 1321-1344, mai./ago. 2021.

https://doi.org/10.22197/rbdpp.v7i2.471

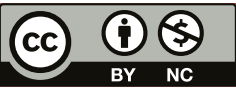

Esta obra está licenciada com uma Licença Creative Commons Atribuição-NãoComercial 4.0 Internacional. 